\title{
ALTERAÇÕES HEPÁTICAS NA ICTERÍCIA OBSTRUTIVA
}

\author{
HEPATIC ALTERATIONS IN THE OBSTRUCTIVE JAUNDICE \\ Orlando de Castro e Silva Jr', Reginaldo Ceneviva ${ }^{1}$, Cláudia Carvalho Rizzo², Ajith Kumar Sankarankutty³, \\ Rubens Gutierrez Granato ${ }^{4}$ \& Luiz Alfredo G. Menegazzo ${ }^{4}$
}

Docente $^{1}$; Médica Anestesiologista2; Aluno do Curso de Pós-Graduação ${ }^{3}$ do Departamento de Cirurgia, Ortopedia e Traumatologia ${ }^{1,2,3}$; Alunos do Curso de Graduação ${ }^{4}$ da Faculdade de Medicina de Ribeirão Preto da Universidade de São Paulo.

CorResPondência: Prof. Dr. Orlando de Castro e Silva Jr. - Disciplina de Gastroenterologia do Departamento de Cirurgia, Ortopedia e Traumatologia da Faculdade de Medicina de Ribeirão Preto da Universidade de São Paulo - Campus Universitário - CEP: 14048-900 Ribeirão Preto - SP.

CASTRO E SILVA Jr. O. et al. Alterações hepáticas na icterícia obstrutiva. Medicina, Ribeirão Preto, 30: 165-172, abr./jun. 1997.

RESUMO: As alterações na função hepática de pacientes com icterícia obstrutiva são descritas nesta revisão, correlacionando-as com a morbidade e a mortalidade operatória. As várias alterações fisiopatológicas que levam ao dano hepatocelular e a disfunção do sistema imune também são analisadas.

UNITERMOS: Colestasia. Fígado. Sistema Imune.

\section{INTRODUÇÃO}

Pacientes com icterícia por obstrução biliar extra-hepática (OBEH) ainda apresentam elevados índices de morbidade e mortalidade pós-operatória ${ }^{1,2,3}$. Além de complicações relacionadas à menor resistência imunológica ${ }^{4,5}$, as alterações hepáticas e sistêmicas, causadas pela impregnação, sobretudo de sais biliares e bilirrubinas, contribuem, de forma significativa, para que complicações pós-operatórias, como sepse, distúrbios hemorrágicos e insuficiência renal, sejam frequientes nesses doentes ${ }^{1,3,6 / 9}$.

Embora o aumento das bilirrubinas séricas seja a alteração bioquímica mais óbvia da icterícia obstrutiva, pela cor amarela característica, causada pela impregnação da pele e dos fluidos orgânicos, o aumento dos níveis séricos e teciduais hepáticos de sais biliares pode ser mais significativo na indução da lesão hepatocelu$\operatorname{lar}^{2,10}$. Adicionalmente, um componente de isquemia funcional, que ocorre em função da diminuição do fluxo portal, pode ser, eventualmente, o fator iniciador do mecanismo da lesão hepática na icterícia obstrutiva ${ }^{11}$.
Isto posto, é necessário que se leve em consideração que um paciente portador de icterícia tem, além de sua pele e escleróticas amarelas, o coração, os rins e, sobretudo, o fígado ictéricos com uma gama de alterações hepatocelulares que podem ser responsáveis, muitas vezes, pelo insucesso da terapêutica cirúrgica.

As múltiplas funções do fígado são vitais para a manutenção da vida. Essas funções incluem a síntese protéica, metabolismo de aminoácidos, carboidratos e lipídios. Alterações em cada uma dessas funções têm sido exaustivamente estudadas na icterícia obstrutiva, situação em que o fluxo biliar está, parcial ou totalmente, interrompido pela obstrução extra-hepática da via biliar ${ }^{1,12,13}$.

A obstrução biliar causa dois problemas mecânicos importantes. Primeiro, substâncias excretadas pelo fígado não chegam ao duodeno. Segundo, a elevação da pressão nos ductos biliares impede a excreção da bile e facilita o seu refluxo para o sangue ${ }^{5,12}$. Assim, as bilirrubinas direta e total, a fosfatase alcalina, ácidos biliares e a gamaglutamiltransferase estão aumentados no plasma, e o urobilinogênio urinário e fecal estão 
diminuídos ou ausentes. Trabalhos mostram que, apesar do aumento sérico e hepático dos sais biliares e bilirrubinas, o aumento dos sais biliares é mais lesivo para o fígado do que as bilirrubinas ${ }^{1,2,5}$.

\section{Sais Biliares}

O aumento da pressão, na via biliar, causa diminuição na síntese de sais biliares e conseqüente diminuição do fluxo biliar - sal biliar dependente ${ }^{-5}$. Os sais biliares, impossibilitados de serem secretados na bile, são excretados na urina, por transporte ativo e por difusão passiva de conjugados sulfatados ${ }^{5}$. A despeito dessa via alternativa de excreção, os níveis séricos e hepáticos estão aumentados na obstrução biliar extra-hepática. Entretanto, por outro lado, o "pool" total de sais biliares diminui tanto pela interrupção da circulação entero-hepática como pela diminuição de sua síntese ${ }^{2,5}$.

A síntese dos ácidos biliares, a partir do colesterol, é catalisada por enzimas do retículo endoplasmático (microssomos). Muitas das reações enzimáticas são catalizadas por enzimas do citocromo P-450. O resultado final dessas reações enzimáticas é a conversão de uma molécula de colesterol altamente insolúvel para uma molécula de ácido biliar solúvel, que possui propriedades detergentes ${ }^{2,5}$.

A disfunção hepática, na icterícia obstrutiva, pode ocorrer pelo aumento dos níveis de ácidos biliares de grande poder detergente, como, por exemplo, o ácido quenodeoxicólico ${ }^{2,3,5,13}$. O aumento da concentração hepática do ácido quenodeoxicólico diminui a atividade do sistema hepatocitário do citocromo P-450, causando alterações no metabolismo dos ácidos biliares, com formação de ácidos biliares atípicos. O nível plasmático de sais biliares pode atingir níveis semelhantes àqueles que, in vitro, demonstraram atividade detergente, e que podem ser responsáveis tanto pelas lesões hepatocelulares como pelo prurido que se observa na colestase extra-hepática. Devido à alta toxicidade do ácido quenodeoxicólico, sua transformação em ácido cólico (menos tóxico) pode ser um mecanismo protetor das lesões do fígado ictérico ${ }^{2}$.

O fluxo biliar - sal biliar independente -, da mesma forma, está diminuído, provavelmente em consequiência do aumento da pressão intracanalicular. Isto resulta em retenção intracelular de bilirrubinas direta e indireta $^{2,5}$.

A interrupção do fluxo biliar causa aumento tanto do colesterol sérico quanto da lipoproteína $X$, e leva à deficiência da aciltransferase, e diminuição das lipases hepáticas, com conseqüente lesão das membranas he- patocelulares $^{2}$. Sabe-se que os ácidos biliares liberam uma substância pruridogênica, que é metabolizada pelo citocromo P-450. Entretanto, a atividade deste citocromo está diminuída na obstrução biliar extra-hepática, o que leva a aumento hepático e sérico da substância pruridogênica, causando prurido cutâneo de maior ou menor intensidade ${ }^{2}$.

\section{Bilirrubinas}

A bilirrubina, no meio intracelular, possui uma variedade de efeitos tóxicos: diminui a captação de oxigênio pelas mitocôndrias hepáticas, apresenta efeito desacoplador da oxidação fosforilativa mitocondrial, e diminui a atividade dos citocromos da cadeia respiratória $^{14}$. Segundo Osawa et al. ${ }^{15,16}$, as bilirrubinas também apresentam ação inibitória tóxica das enzimas oxidativas (nicotinamida, adenina, nucleotídeo oxidase e succinato oxidase) devido às altas concentrações intracitoplasmáticas de bilirrubinas. Com relação ao efeito desacoplador das bilirrubinas sobre a oxidação fosforilativa mitocondrial ${ }^{17}$, este fenômeno não foi verificado por outros autores ${ }^{18 / 21}$. Mitocôndrias hepáticas de ratos ictéricos, submetidas a dois estímulos consecutivos com ADP, mantiveram a razão de controle respiratório sem desacoplamento da oxidação fosforilativa ${ }^{18}$. Daccach et al. ${ }^{21}$ verificaram que a ação lesiva das bilirrubinas sobre mitocôndrias esplênicas ocorreram apenas quando se induziu, previamente, isquemia normotérmica às células esplênicas, mostrando que a isquemia agiu como fator desencadeante das lesões induzidas pelas bilirrubinas.

Adicionalmente, trabalhos mostram alterações no metabolismo intermediário hepático na OBEH, devidas à isquemia funcional do fígado ${ }^{11}$. O fígado que, em condições normais, é glicogenético ${ }^{11}$, durante a OBEH transforma-se em glicogenolítico ${ }^{11}$, perdendo, assim, a capacidade de armazenar glicogênio. Esta mudança de padrão metabólico ocorre em função da ativação da piruvatoquinase, enzima alostérica, chave no metabolismo da glicose ${ }^{11}$.

Assim, alterações no metabolismo energético do fígado, na OBEH, podem resultar do acúmulo de sais biliares tóxicos e bilirrubinas e seus metabólitos ${ }^{15,16}$, ou da isquemia funcional que se instala nessa condição ${ }^{11}$.

Osawa et al. ${ }^{15,16}$, descreveram dois padrões de tolerância à glicose em animais de experimentação e em humanos com icterícia obstrutiva. Esses padrões distintos estão relacionados com o grau de alteração produzido na atividade fosforilativa mitocondrial hepática. Pacientes que apresentam padrão linear de 
intolerância à glicose (níveis de glicemia permaneceram elevados por um período maior do que cento e vinte minutos após a administração de glicose, apresentaram maior mortalidade pós-operatória do que pacientes com padrão parabólico de tolerância à glicose (aumento inicial da glicemia e normalização aos cento e vinte minutos). Estas alterações do metabolismo energético do fígado, descritas acima, podem explicar a tendência de os pacientes com OBEH apresentarem curvas glicêmicas diabetóides após sobrecarga oral ou endovenosa de glicose.

\section{Isquemia Funcional}

Vários autores verificaram diminuição efetiva do fluxo portal na obstrução biliar extra-hepática. Estudos experimentais, em ratos e cães, mostraram que essa queda no fluxo portal chega a níveis de 40 a $50 \%{ }^{11,22,23}$. Essa diminuição do fluxo portal não chega a ser totalmente compensada por aumento do fluxo arterial hepático, o que determina diminuição do fluxo sanguíneo hepático total ${ }^{23}$.

Estudos experimentais seriados, em cães, mostraram redução de $36 \%$ no fluxo arterial e $44 \%$ no fluxo portal, após indução da obstrução biliar extrahepática $^{23}$. Adicionalmente, verificou-se aumento de
200\% na resistência venosa portal intra-hepática, sem aumento similar na resistência arterial ${ }^{23}$. Outros estudos mostraram que cães com obstrução biliar extra-hepática desenvolveram hipertensão portal sinusoidal e grande número de "shunts" portossistêmicos ${ }^{24}$.

Reuter \& Chuang, em $1976^{25}$, em trabalho medindo pressões da veia supra-hepática livre e ocluída e da veia porta, sugeriram que o local primário de resistência ao fluxo era pré-sinusoidal. Outros estudos, ainda na década de setenta, mostraram que a dilatação dos ductos biliares intra-hepáticos provocam o aumento da pressão portal de forma pós-sinusoidal ${ }^{6}$. Em 1991, estudos experimentais mostraram que o fluxo portal, reduzido durante a obstrução biliar extra-hepática, aumentava significativamente, após três minutos de drenagem das vias biliares, confirmando a relação de causa e efeito entre a dilatação ductal e a redução do fluxo portal ${ }^{13}$.

Esta diminuição do fluxo sanguíneo hepático predispõe o órgão a estados de anoxia ou mesmo de isquemia funcional ${ }^{11,13}$, que podem ser o fator iniciador das lesões celulares, tornando os hepatócitos e suas organelas mais sensíveis aos efeitos tóxicos dos sais biliares e bilirrubinas, que, "per si”, não seriam lesivas as células hepáticas (Figura 1).

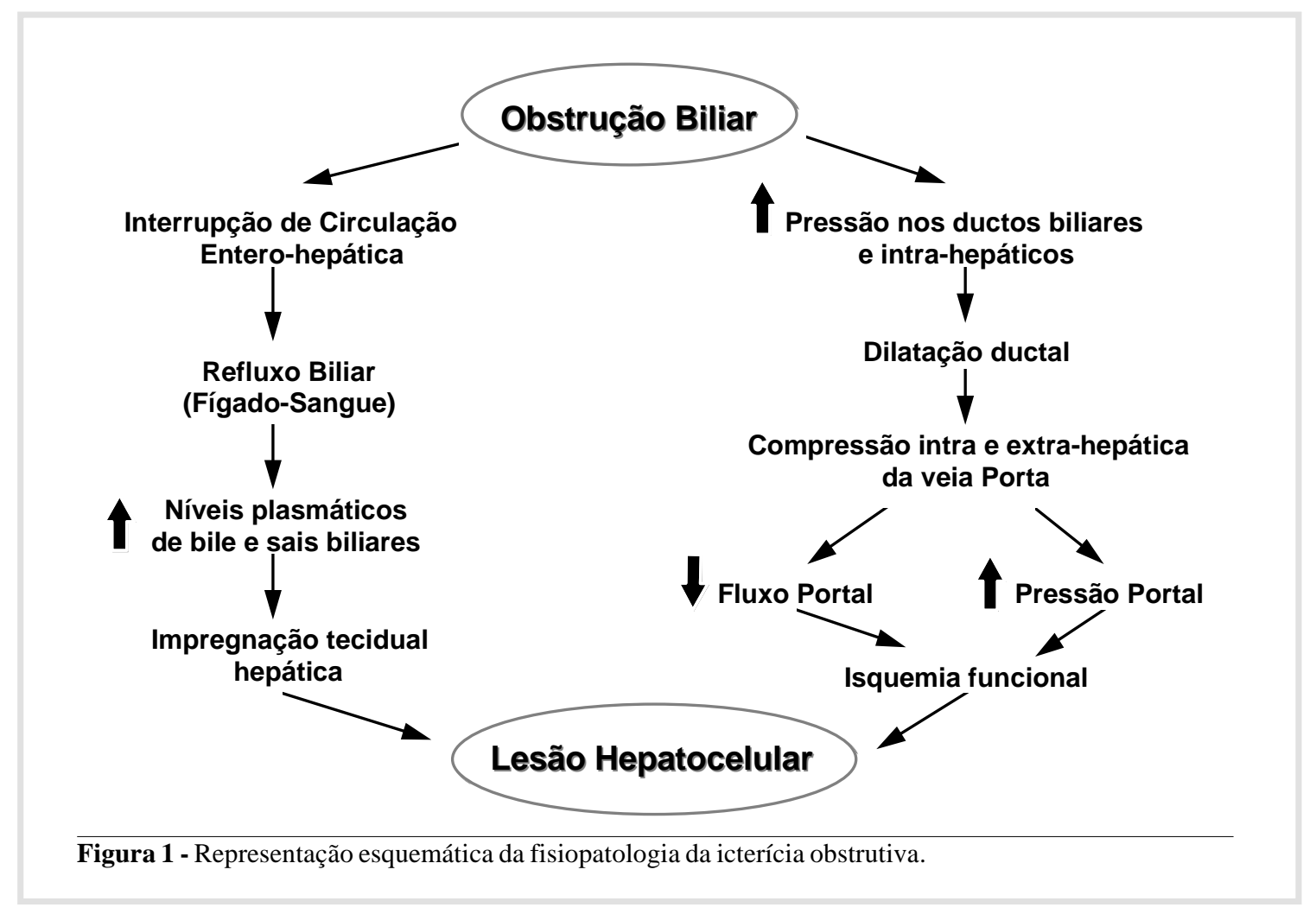


Por outro lado, estudos mostram que o fígado, na icterícia obstrutiva, pela diminuição do fluxo portal torna-se funcionalmente dependente do sangue arterial hepático ${ }^{18,26,27}$.

Ratos ictéricos apresentaram valores elevados de aminotransferases (ALT e AST) e necrose hepatocelular, após a ligadura da artéria hepática ${ }^{27 / 31}$. Isto su- gere que o fígado ictérico trabalha numa condição limite de necessidade e suprimento de energia ${ }^{32 / 34}$. Qualquer condição adversa, que diminua a oferta de oxigênio ao fígado, como choque circulatório, hipovolemia e desidratação, induz lesões hepatocelulares adicionais àquelas já causadas pela impregnação de sais biliares e bilirrubinas (Figura 2).

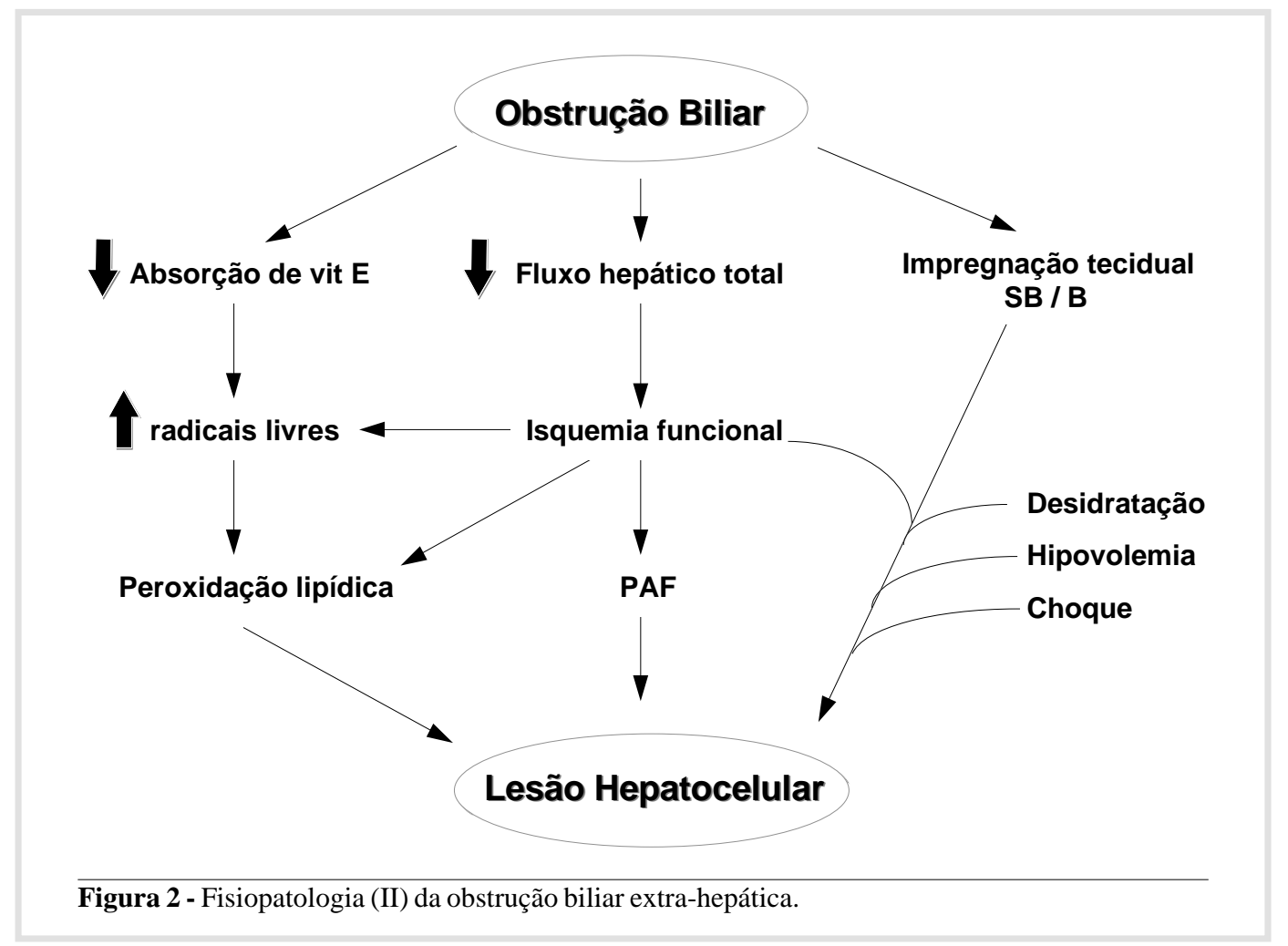

Trabalhos, na literatura, mostraram que, após a descompressão das vias biliares dilatadas, houve redução brusca da pressão da veia hepática ocluída (PVHO), pressão venosa portal e pressão arterial e venosa central, mostrando que, nos primeiros minutos até uma hora após a descompressão das vias biliares, houve um sequestro de líquidos no interior do fígado. Isto causou diminuição do volume plasmático efetivo, com conseqüente hipotensão $0^{8,35}$. Clinicamente deve-se prever o potencial para alterações hemodinâmicas e sequestro de grandes volumes de líquido nos espaços perivasculares intra-hepáticos, após descompressão biliar, para que se possa fazer o tratamento adequado no período perioperatório, através de hidratação adequada ${ }^{36}$.

A possível explicação fisiopatológica para o sequestro significativo de líquidos no fígado, após a dre- nagem das vias biliares (DVB) é a lesão das membranas celulares causada tanto pelas lipases endógenas e pelos radicais livres de oxigênio, aumentados no tecido hepático durante a obstrução biliar extra-hepática (Figura 2) como pela isquemia funcional que se instala nessa condição ${ }^{11,37}$. Após a DVB, com aumento do fluxo portal e arterial hepático, haveria lesões celulares devido à reperfusão hepática, colocando em comunicação os diversos compartimentos teciduais do fígado (intracelular, intravascular e intersticial) ${ }^{35}$.

O colesterol, triglicerídeos e lecitina estão elevados na OBEH. Alterações estruturais, no retículo endoplasmático, induzem ao aumento da LP-X (lipoproteína $X)$, potencialmente lesiva às membranas hepatocelulares $^{2}$. Os sais biliares, aumentados no tecido hepático, solubilizam os componentes da membrana ca- 
nalicular, particularmente os fosfolipídeos, levando a aumento plasmático de lipídios oriundos das membranas hepatocelulares ${ }^{2,3}$. A solubilização de membranas permite a liberação, para o sangue, de ectoenzimas como a fosfatase alcalina ${ }^{38}$. Estudos das isoenzimas da fosfatase alcalina mostram que uma fração se origina da membrana citoplasmática e as outras, do citoplas$\mathrm{ma}^{2,38}$. A fosfatase alcalina de membrana é a principal fosfatase protéica da membrana citoplasmática, e algumas das disfunções hepatocelulares podem ser resultantes da perda da atividade desta enzima ${ }^{2}$.

Estudos recentes descrevem aumento dos níveis hepatocelulares de substâncias oxidantes como o malonaldeído, durante a obstrução biliar extra-hepática, que podem ocorrer tanto pela diminuição dos níveis hepáticos de vitamina $\mathrm{E}^{38}$ (menor absorção a nível intestinal, pela ausência ou diminuição de sais biliares intraluminares) e impregnação hepática dos sais biliares e bilirrubinas ${ }^{2,5}$, como pela isquemia funcional ${ }^{11}$. Da mesma forma, durante a OBEH, constatou-se diminuição significativa de enzimas hepáticas antioxidantes como o glutation, a glutation peroxidase e glutation transferase ${ }^{37}$. Assim, na OBEH, a redução dessas enzimas hepáticas, antioxidantes, implica no aumento tecidual de radicais livres, altamente lesivos às membranas hepatocelulares pela peroxidação lipídica a que elas induzem ${ }^{37}$.

\section{SÍNTESE PROTÉICA E FATORES DE COAGULAÇÃO}

O fígado ocupa papel chave na síntese protéica. A albumina é, quantitativamente, a proteína plasmática mais importante sintetizada pelo fígado. Entretanto, pela sua vida média longa (vinte, vinte e seis dias) detectam-se apenas discretas alterações nessa proteína, causadas pela lesão hepatocelular ${ }^{2,3}$. Mesmo assim, diversos estudos têm demonstrado que níveis baixos de albuminemia representam risco operatório significativo em pacientes portadores de obstrução biliar extra-hepática ${ }^{27}$. Como indicador do nível da síntese protéica pelo fígado, a pré-albumina é mais sensível, posto que sua vida média na circulação sanguínea é de um, nove dias ${ }^{2,3}$.

Outro aspecto importante da síntese de proteínas relaciona-se com o papel do fígado na manutenção do processo de coagulação. Desta forma, o fibrinogênio, a protrombina e os fatores V, VII, IX e X podem estar afetados na icterícia obstrutiva de longa duração. Alterações na coagulação, em muitas casos de obstru- ção biliar extra-hepática, devem-se à insuficiência de absorção das vitaminas lipossolúveis A, D e K, pela ausência de sais biliares no intestino. Em consequiência da má absorção da vitamina $\mathrm{K}$, o tempo de protrombina (TP) pode estar aumentado, levando à hemostasia deficiente, em pacientes com $\mathrm{OBEH}^{39}$. Assim, na icterícia obstrutiva o processo de coagulação pode normalizar-se com administração parenteral de vitamina $\mathrm{K}$.

\section{ALTERAÇÕES IMUNOLÓGICAS}

A sepse é uma das principais causas de morbidade e mortalidade em pacientes com OBEH. Septicemia por gram-negativos é freqüente após manipulação da via biliar obstruída, e pode evoluir para choque séptico com insuficiência de múltiplos órgãos. Endotoxemia ocorre no pré-operatório, em 20 a $65 \%$, e, em aproximadamente $50 \%$, no pós-operatório de pacientes com $\mathrm{OBEH}^{2.40,41}$.

A endotoxemia portal foi encontrada em aproximadamente metade dos pacientes com OBEH. Trabalhos mostraram associação entre a endotoxemia pré-operatória e a mortalidade no pós-operatório ${ }^{2}$. A incidência de endotoxemia não se correlaciona com os níveis de bilirrubina e encontra-se diretamente relacionada com a duração da icterícia ${ }^{42}$.

A ausência ou diminuição de bile no intestino delgado leva à perda do efeito emulsificante e antiendotóxico dos sais biliares, provocando níveis elevados de endotoxinas, no intestino grosso, que serão absorvidas através da circulação portal ${ }^{43,44}$.

Há evidências de que a OBEH causa translocação bacteriana da luz intestinal para os linfonodos mesentéricos e inibição parcial da função fagocítica do sistema retículoendotelial hepático e que bactérias, endotoxinas e imunocomplexos não são depurados de forma eficiente pelo fígado ictérico ${ }^{45}$. As células de Kupffer constituem mais de $85 \%$ do sistema fagocítico mononuclear (SFM ${ }^{46}$, estrategicamente localizadas na confluência da drenagem venosa portal. Estas células, altamente especializadas, possuem a responsabilidade da defesa do hospedeiro em sequestrar e eliminar antígenos, endotoxinas e microorganismos oriundos do trato gastrointestinal ${ }^{47}$. A exposição das células de Kupffer às endotoxinas presentes no sangue portal faz com que essas células produzam grandes quantidades de PAF - fator de ativação plaquetária -importante como indutor de lesões hepatocelulares. A translocação bacteriana e a disfunção do SFM podem ser responsáveis pela alta incidência de complicações sépticas em pacientes com icterícia obstrutiva. 
A inibição fagocítica dos macrógafos hepáticos e diminuição da atividade das células de Kupffer devem-se, provavelmente, ao aumento da pressão biliar intraductal e ao acúmulo de sais biliares e bilirrubinas no sangue e no tecido hepático. Recentemente, a liberação de mediadores específicos, como TNF- $\alpha$ (Fator de necrose tumoral, liberado pelos macrófagos), interleucinas, e leucotrienos, poderia estar correlacionadas com a atividade fagocítica das células de Kupffer. $\mathrm{Na}$ OBEH, o TNF- $\alpha$ estaria diretamente envolvido nas alterações hepatocelulares ${ }^{48}$. A esplenomegalia, verificada em alguns casos de OBEH prolongada, também pode ser devida à inibição do SFM hepático e esplênico (Figura 3).

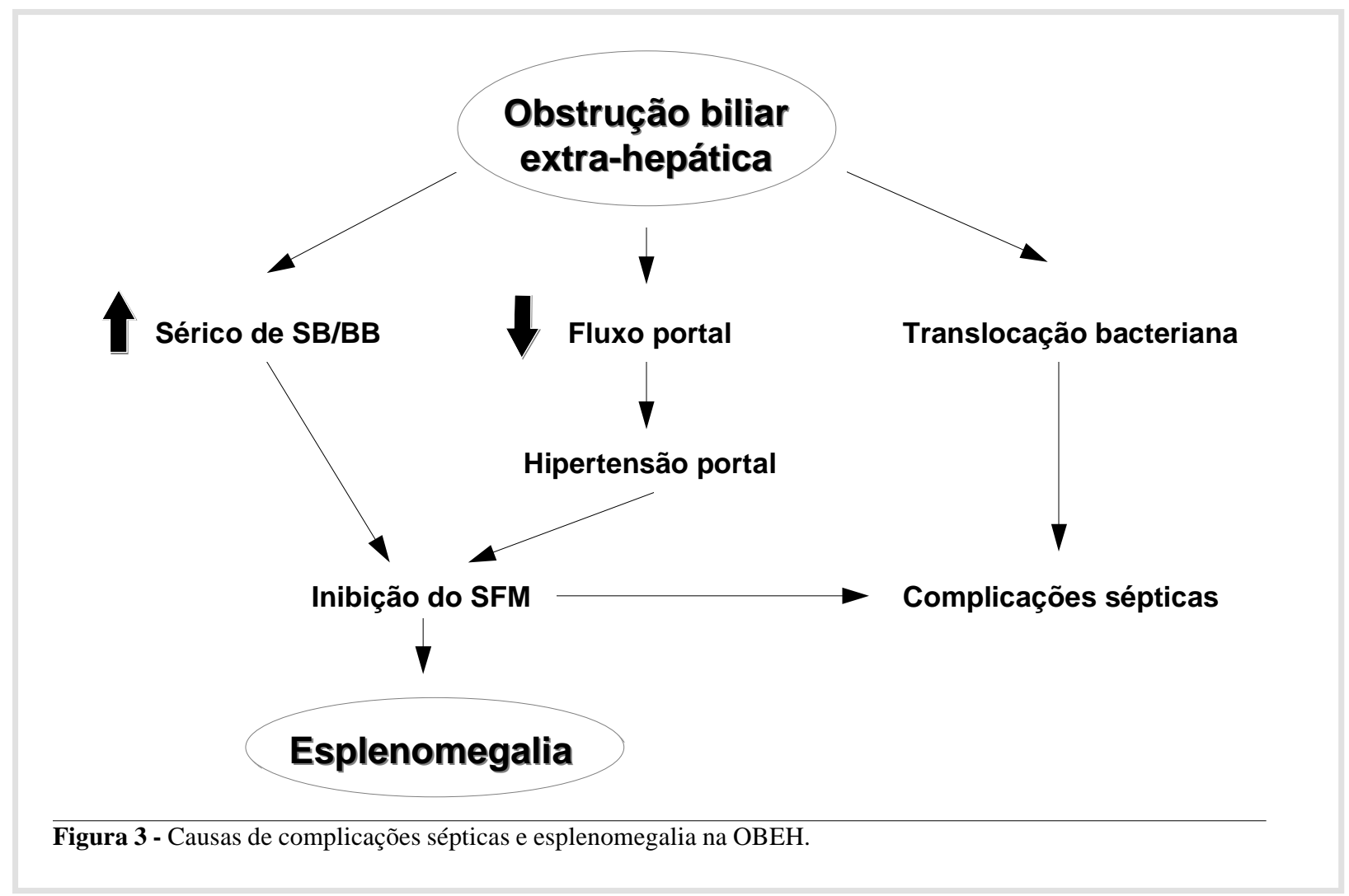

\section{Considerações finais}

Os efeitos da obstrução biliar extra-hepática sobre o fígado, descritos acima e resumidos na Tabela I, predispõem os pacientes ictéricos a elevado risco operatório. Entretanto, mesmo com os testes de função hepática de que dispomos atualmente, é difícil avaliar, no pré-operatório, o grau de reserva funcional do fígado que se encontra diminuído na OBEH ${ }^{11}$.

Assim, é necessário que medidas preventivas sejam tomadas, para que fatores como desidratação, hipovolemia, choque e infecção não venham, no perioperatório, agravar as condições hepáticas desses doentes. Desta forma, é possível diminuir os índices de morbidade e mortalidade pós-operatória na $\mathrm{OBEH}$.
Tabela I - Alterações hepáticas na OBEH

1. Diminuição da síntese protéica

2. Diminuição da síntese de fatores de coagulação

3. Diminuição da fração microssomal P-450

4. Aumento da pressão portal

5. Diminuição do fluxo portal

6. Diminuição do fluxo arterial hepático

7. Aumento do refluxo colangiovenoso e colangiolinfático

8. Disfunção mitocondrial

9. Aumento dos radicais livres intra-hepáticos

10. Aumento do fator de ativação plaquetária (PAF)

11. Diminuição do glicogênio hepático

12. Inibição do sistema fagocítico mononuclear

13. Aumento do TNF - alfa 
CASTRO E SILVA Jr. O. et al. Hepatic alterations in the obstructive jaundice. Medicina, Ribeirão Preto, 30: 165-172, apr./june 1997.

ABSTRACT: The alterations in the hepatic function of patients with obstructive jaundice are described in this chapter, correlating them with the operative morbidity and mortality. The various pathophysiologic alterations the lead to hepatocelular damage and the immune system disfunction are analysed as well.

UNITERMOS: Cholestasis. Liver. Immune System.

\section{REFERÊNCIAS BIBLIOGRÁFICAS}

1 - O'CONNOR MJ. Mechanical biliary obstruction. Am Surg 51: 245-251, 1985

2 - REICHEN J \& SIMON FR. Cholestasis. In: ARIAS IM et al. The liver: biology and pathobiology. 3 th ed. Raven Press, New York, p. 1291-1326, 1994.

3 - SCHAFFNER F. Cholestasis. In: SADLER GHM; WRIGHT R \& ARTHUR MJR. Liver and biliary disease. 3th ed. W.B. Saunders, London, p. 371-396, 1992.

4 - ADACHI Y. et al. Hepatic macrophage malfunction in rats with obstructive jaundice and its biological significance. J Hepatol 171-176, 1992.

5 - SCOTT-CONNER CEH \& GROGAN JB. The pathophysiology of biliary obstruction and its effect on phagocytic and immune function. J Surg Res 57: 316-336, 1994.

6 - MATHIE RT; NAGORNEY DM \& BLUMGAST LH. Flujo sanguíneo hepático fisiologia, medicion e importância clínica. In: BLUNGART LH. Cirurgia del fígado y de las vias biliares. Medica Panamericana, Argentina, p. 87-103, 1990.

7 - MURIEL P et al. Protective effect of S-adenosyl-L-nethionine on liver damage induced by biliary obstruction in rats: a histological, ultrastructural and biochemical approach. J Hepatol 21: 95-102, 1994.

8 - STERR ML et al. Chronic biliary obstruction: hemodynamic effects of decompression. Surg Forum 19: 342-344, 1968.

9 - WAIT RB \& KAHNG KU. Renal failure complication obstrutive jaundice. Am J Surg 157: 256-263, 1989.

10 - VITALE GC et al. Reversible bile acid changes in bile duct obstruction and its potential for hepatocellular injury. $\mathbf{J}$ Hepatol 14: 151-156, 1992.

11 - ROSELINO JE; CASTRO E SILVA JR. O \& CENEVIVA R. Lack of control of liver gluconeogenesis in cholestatic rats with reduced portal blood flow. Hepatology 16: 1055-1060, 1992.

12 - LEE RG. Cholestasis and biliary obstruction. In: LEE RG, ed. Diagnostic liver pathology. Mosby, St. Louis, p. 82-107, 1994.

13 - CASTRO E SILVA JR. O. Fluxo portal e atividade enzimática da piruvato quinase na obstrução biliar extra-hepática. Tese Livre Docência, Faculdade de Medicina de Ribeirão Preto da USP, Ribeirão Preto, p. 1-87, 1991.
14 - QUENEAU PE \& MONTET JC. Hepatoprotection by hydrophilic bile salts. J Hepatol 21: 260-268, 1994.

15 - OSAWA K et al. Significance of glucose tolerance as prognostic signs in hepatectomized patient. Am J Surg 131: 541-546, 1976.

16 - OSAWA K et al. Oral glucose tolerance in patients with jaundice. Surg Gynecol Obstet 140: 582-588, 1975.

17 - NOIR B et al. Bilirrubin: a multi-site inhibitor of mitochondrial respiration. Febs Lett 27: 270-274, 1972.

18 - CASTRO E SILVA JR O et al. Alterações não desacopladoras da fosforilação oxidativa mitocondrial na colestase extra-hepática. Acta Cir Bras 4: 102-105, 1989.

19 - CASTRO E SILVA JR., O et al. Integrity of liver inner mitochondrial membrane in rats with extrahepatic cholestasis. Braz J Med Biol Res 24: 167-170, 1991.

20 - CRUZ CAT et al. Função mitocondrial esplênica na obstrução biliar extra-hepática. Acta Cir Bras 8: 8-10, 1993.

21 - DACCACH $V$ et al. Função mitocondrial esplênica na vigência de obstrução biliar extra-hepática e isquemia do baço. Medicina, Ribeirão Preto 27: 421-422, 1994.

22 - HUNT DR. Changes in liver blood flow with development of biliary obstruction in the rat. Aust N Z J Surg 49: 733737, 1979.

23 - MATHIE RT et al. Hepatic hemodinamics after chronic obstruction of the biliary tract in the dog. Surg Gynecol Obstet 166: 125-130, 1988.

24 - BOSCH J et al. Chronic bile duct legation in the dog: hemodynamic characterization of a portal hypertensive model. Hepatology 3: 1002-1007, 1983.

25 - REUTER SR \& CHUNG VP. The location of increased resistence to portal blood flow in obstructive jaundice. Invest Radiol 11: 54-59, 1976.

26 - SOARES AF et al. Effect of hepatic artery ligation in rats with chronic extrahepatic cholestasis. Braz J Med Biol Res 23: 543-545, 1990.

27 - SOARES AF et al. Biochemical and morphological changes of the liver after hepatic artery ligation in the presence and absence of extrahepatic cholestasis. Int J Exp Pathol 74: 367-370, 1993. 
28 - CASTRO E SILVA Jr O et al. Hepatic morphology changes after hepatic artery ligation in extrahepatic cholestasis. Braz J Med Biol Res 25: 353-355, 1992.

29 - CASTRO E SILVA Jr O et al. Alterações agudas hepatocelulares após ligadura da artéria hepática em ratos com colestase extra-hepática. Acta Cir Bras 5: 55-58, 1990.

30 - CASTRO E SILVA Jr O et al. Impaired liver regeneration in extrahepatic cholestatic rats. Res Surg 4: 32-34, 1992.

31 - SOARES AF et al. The effect of hepatic artery ligation on liver morphology in rats. Res Surg 5: 17-19, 1993.

32 - CASTRO E SILVA JR O et al. Alterações hepáticas e níveis séricos de aminotransferases 24 horas após ligadura do ducto hepático em ratos. Acta Cir Bras 6: 159-161, 1991.

33 - KONO N \& NAKANUMA Y. Ultrastructural and immunohistochemical studies of the intrahepatic peribiliary capillary plexus in normal livers and extrahepatic biliary obstruction in human beings. Hepatology 15: 411-418, 1992.

34 - SOUZA MEJ et al. Serum transaminase levels in the acute phase of chronic extrahepatic cholestasis. Braz J Med Biol Res 23: 995-997, 1990.

35 - TAMAKUMA $S$ et al. Relationship between hepatic hemogynamics and biliary pressure in dogs its significance in clinical shock following biliares decompression. Jpn $\mathbf{J}$ Surg 5: 255-268, 1975.

36 - RIZZO CC \& CASTRO E SILVA JR., O. Anestesia em paciente com icterícia obstrutiva: relato de caso. Medicina, Ribeirão Preto 27: 242-245, 1994.

37 - SINGH S et al. Antioxidant defenses in the bile duct-ligated rat. Gastroenterology 103: 1625-1629, 1992.

38 - KAPLAN MM \& RIGHETTI A. Induction if rat liver alkaline phosphatase: the mechanism of the serum elevation in bile duct obstruction. J Clin Invest 49: 508-516, 1970.
39 - MAZE M. Anesthesia and the liver. In: MILLER R. ed. Anesthesia. Churchill Livingstone, Edinburgh, p. 1969-1980, 1994.

40 - PELLEGRINI CA. Pathophysiology of biliary obstruction. In: WAY LW \& PELLEGRINI CA. Surgery of the Gallbladder and bile ducts. W.B. Saunders, Philadelphia, p. 103-117, 1987.

41 - TANAKA N et al. Impaired liver clearence of bacteria in rats with chronic biliary obstruction. Res Exp Med 185: 173-179, 1985.

42 - CLEMENTS B et al. Effects of extrahepatic jaundice on kupffer cell clearance capacity. Arch Surg 128: 200-205, 1993.

43 - BAILEY ME. Endotoxin, bile salts and renal function in obstructive jaundice. Br J Surg 66: 392-397, 1976.

44 - KOSCAR LT; BERTOL L \& VARTEREZ V. Effect of bile acids on intestinal absorption of endotoxin in rats. $\mathbf{J}$ Bacteriol 100: 220-223, 1969.

45 - DING JW et al. Inhibition of bacterial translocation in obstructive jaundice by muramyl tripeptide phosphatidylethanolamine in the rat. J Hepatol 20: 720-728, 1994.

46 - BIOZZI G \& STIFFER C. The pathophyssiology of the retrarbendothelial cells of the liver and spleen. In: POPER H et al. Progress in liver disease. Grune \& Stratton, New York, p. 166-191, 1965.

47 - JONES A et al. Tumour necrosis factor, cholestatic jaundice, and chronic liver disease. Gut 31: 938-939, 1990.

48 - ZHOU W et al. Role of platelet-activating factor in hepatic responses after bile duct ligation in rats. Am J Physiol 263: 6587-6592, 1992.

Recebido para publicação em 05/05/97

Aprovado para publicação em 28/05/97 